\title{
Orbital Myositis Presenting as Painless Ophthalmoplegia
}

\author{
Akiyuki Hiraga, Akiyuki Uzawa and Ikuo Kamitsukasa
}

Key words: orbital myositis, computed tomography, magnetic resonance imaging

\section{(DOI: 10.2169/internalmedicine.46.0108)}
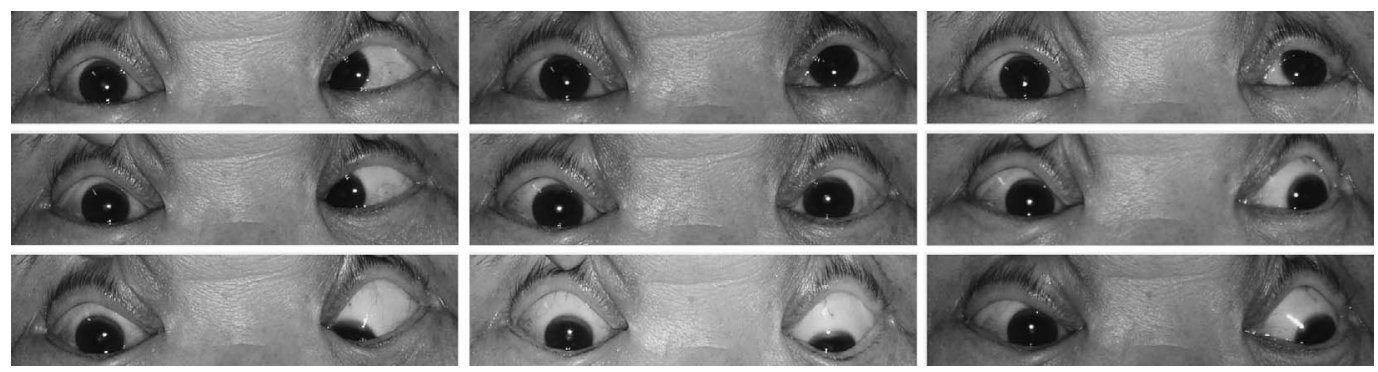

Picture 1. Nine cardinal positions of gaze, obtained on admission. There is moderate total limitation of the right eye, especially abduction.
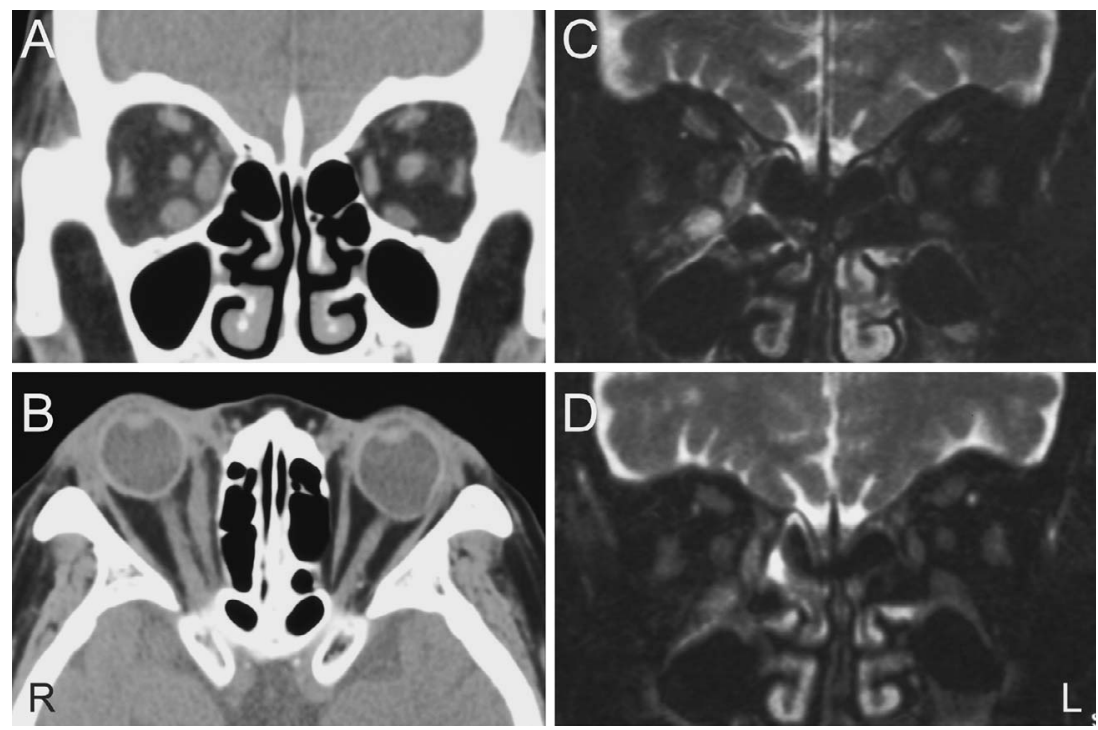

Picture 2. (A) Coronal and (B) axial computed tomography scan of the orbits before treatment shows gross thickening of the right medial rectus and inferior rectus muscles, and the superior oblique muscle. Axial scan also shows exophthalamous. (C) Coronal fat-suppression T2-weighted magnetic resonance imaging scan of the orbits before and (D) 45 days after corticosteroid treatment. There is lessening of the thickness and high signal of these muscles.

A 78-year-old non-diabetic woman was admitted to our hospital with a 20-day history of acute painless right ptosis, upper eyelid swelling, and diplopia. There were moderate total limitations of eye movement (Picture 1). Computed tomography and magnetic resonance images showed gross en- largement of the belly of the extraocular muscles (Picture 2). A high signal was present in these muscles on T2weighted imaging. There was no clinical or serological evidence of collagen vascular disease, sarcoidosis, or Graves' disease. Idiopathic orbital myositis (IOM) was diagnosed. 
Corticosteroid treatment produced dramatic improvement.

IOM is a nonspecific orbital inflammation primarily involving the extraocular muscles. Its cardinal clinical feature is orbital pain exacerbated by eye movement (1). IOM is one of the most important differential diagnoses of "painful ophthalmoplegia", ranking with Tolosa-Hunt syndrome or vascular conditions such as an aneurysm. Painlessness (2) indicates that IOM should not be ruled out in the case of "painless ophthalmoplegia."

\section{References}

1. Scott IU, Siatkowski RM. Idiopathic orbital myositis. Curr Opin Rheumatol 9: 504-512, 1997.

2. Pagès M, Malanda G, Mayembo MA. Ophtalmoplégie indolore et récidivante révélatrice d'une myosite orbitaire. Rev Neurol (Paris) 161: 224-225, 2005

\footnotetext{
(C) 2007 The Japanese Society of Internal Medicine http://www.naika.or.jp/imindex.html
} 